\title{
BMJ Open Navigating choice in the face of uncertainty: using a theory informed qualitative approach to identifying potential patient barriers and enablers to participating in an early phase chimeric antigen receptor $\mathrm{T}(\mathrm{CAR}-\mathrm{T})$ cell therapy trial
}

\author{
Gisell Castillo (10 ,1,2 Manoj M Lalu (1) , , ${ }^{1,2,3}$ Sarah Asad, ${ }^{1,2}$ Madison Foster, ${ }^{1,2}$ \\ Natasha Kekre, ${ }^{2,4}$ Dean A Fergusson (D) , 1,2,5 Terry Hawrysh, ${ }^{6}$ Harold Atkins, ${ }^{2,4}$ \\ Kednapa Thavorn, ${ }^{2,5,7}$ Joshua Montroy, ${ }^{1,2}$ Stuart Schwartz, ${ }^{6}$ Robert A Holt, ${ }^{8}$ \\ Raewyn Broady, ${ }^{9}$ Justin Presseau ${ }^{1,2,5}$
}

To cite: Castillo G, Lalu MM, Asad S, et al. Navigating choice in the face of uncertainty: using a theory informed qualitative approach to identifying potential patient barriers and enablers to participating in an early phase chimeric antigen receptor T (CAR-T) cell therapy trial. BMJ Open 2021;11:e043929. doi:10.1136/ bmjopen-2020-043929

- Prepublication history and additional material for this paper is available online. To view these files, please visit the journal online (http://dx.doi.org/10. 1136/bmjopen-2020-043929).

Received 17 August 2020 Revised 02 February 2021 Accepted 16 February 2021

Check for updates

(c) Author(s) (or their employer(s)) 2021. Re-use permitted under CC BY-NC. No commercial re-use. See rights and permissions. Published by BMJ.

For numbered affiliations see end of article.

Correspondence to Dr Justin Presseau; jpresseau@ohri.ca

\section{ABSTRACT}

Objectives Bench to bedside translation of groundbreaking treatments like chimeric antigen receptor $\mathrm{T}$ (CAR-T) cell therapy depends on patient participation in early phase trials. Unfortunately, many novel therapies fail to be adequately evaluated due to low recruitment rates, which slows patient access to emerging treatments. Using the Theoretical Domains Framework (TDF), we sought to identify potential patient barriers and enablers to participating in an early phase CAR-T cell therapy trial. Design We used qualitative semistructured interviews to identify potential barriers and enablers to patients' hypothetical participation in an early phase CAR-T cell therapy trial. We used the TDF and directed content analysis to identify relevant domains based on frequency, relevance and the presence of conflicting beliefs. Participants Canadian adult patients diagnosed with haematological malignancies.

Results In total, we interviewed 13 participants (8 women, 5 men). Participants ranged in age from 18 to 73 (median=56) and had been living with haematological cancer from a few months to several years. We found participants were unfamiliar with CAR-T cell therapy but wished to know more about treatment safety, efficacy and trial logistics (domains: knowledge, beliefs about consequences). They were motivated by altruistic considerations, though many prioritised personal health benefits despite recognising the goals (ie, establishing safety) of early phase clinical trials (domains: goals, intentions). Every participant valued receiving medical advice from their haematologists and oncologists, though some preferred impartial medical experts to inform their decision making (domain: social influences). Finally, participants indicated that improving access to financial and social supports would improve their trial participation experience (domain: environmental context and resources).

\section{Strengths and limitations of this study}

- We sought to identify implementation barriers to trial participation in advance of a chimeric antigen receptor $\mathrm{T}(\mathrm{CAR}-\mathrm{T})$ cell therapy trial to optimise recruitment strategies.

- We used a comprehensive, evidence-based framework (Theoretical Domains Framework) to identify potential barriers and enablers to trial participation.

- Patient voices were prioritised by ensuring patient views were sought out prior to trial launch and by involving patient partners in study design and analysis.

- Study participants were self-selected and may not fully represent the diversity of haematological cancer patients who may be eligible for a CAR-T cell therapy trial.

- Study results represent anticipated barriers and enablers based on participants' consideration of hypothetically participating in a CAR-T cell therapy trial.

Conclusion Using the TDF allowed us to identify factors that might undermine participation to a CAR-T cell therapy trial and to optimise recruitment processes by considering patient perspectives to taking part in early phase trials. Trial regestration: NCT03765177; Pre-results.

Chimeric antigen receptor $\mathrm{T}$ (CAR-T) cell therapy is a promising new treatment for people with relapsed or refractory B cell leukaemia and lymphoma. ${ }^{12} \mathrm{~A}$ systematic review of early phase trials assessing the safety and efficacy of CAR-T cell therapy found that $77 \%$ of patients with acute lymphocytic 
leukaemia and $54 \%$ of patients with other haematological malignancies that were treated with CD19 targeted CAR-T cells experienced a complete response. ${ }^{3}$ Efforts to refine production and improve toxicity management are ongoing but require further evaluation through rigorous early phase testing. ${ }^{4}$ Unfortunately, recruitment to adult cancer clinical trials remains a challenge, with participation rates ranging between $2 \%$ and $7 \% .^{5-7}$

Given the complex, resource-intensive and intricate nature of CAR-T cell therapy, recruitment strategies must be optimised to promote the timely evaluation of this novel therapy. Past research on patient barriers to participating in cancer clinical trials has underscored treatment arm preference, side effects, uncertain outcomes and the burden of participation, as key barriers to clinical trial participation while enablers have included expecting benefits, perceived low risk, altruistic considerations and hearing about trials from trusted physicians. ${ }^{6-12}$ Identified barriers and enablers that are specific to early phase cancer trials have included narrow eligibility criteria, design concerns, safety and toxicity issues, and patient refusals. ${ }^{13-15}$ Patient reasons for declining participation have included fear of side effects, expecting little benefit, declining health and being in good health. ${ }^{16}$ Motivations for participating in early phase trials include expecting health benefits, the absence of alternative treatment options and hearing about the trial from trusted healthcare providers. ${ }^{17-21}$

The role of physicians in encouraging trial participation has also been emphasised as an important factor as physicians may provide biased or unclear explanations of trial details. ${ }^{82-24}$ One study found that physicians who successfully recruited patients into trials were more likely to provide information regarding trial benefits, address patient concerns, explain side effects and offer resources to manage patient anxiety than those who were less successful at recruiting patients. ${ }^{23}$ Physicians have also identified barriers to screening for early phase clinical trials, including concerns over safety, narrowly defined inclusion criteria, and time and resource constraints (see Castillo et al. 2021. Hematologists' barriers and enablers to screening and recruiting patients to a Chimeric Antigen Receptor (CAR) T cell therapy trial: A theory informed interview study; forthcoming). Though some work has begun to explore how to optimise strategies for improving trial recruitment by drawing on theory, ${ }^{25-27}$ most strategies for improving trial recruitment rates are seldom theoretically driven. ${ }^{25}$ Instead, many interventions focus on providing participants with information regarding clinical trials using various modalities (eg, video, website) and have had moderate or inconsistent effects on subsequent trial participation. ${ }^{28-31}$ This indicates a need for designing novel, fit-for-purpose strategies that go beyond information provision to ones that harness the cumulative evidence base associated with theory-informed approaches. ${ }^{32}$

The Theoretical Domains Framework (TDF) was developed to synthesise the key factors across theories of behaviour and behaviour change into 12 domains. ${ }^{33}$ The framework was then validated and expanded into 14 domains. ${ }^{34}$ The domains include factors known to affect decisions and actions and can be used to probe which barriers and enablers in which domains might be influencing a particular decision. The TDF is distinct from other approaches in that its breadth facilitates identification of particular anticipated barriers/enablers for a given trial and sets the stage for linking barriers and enablers to strategies that are consistent with behavioural change theory and tailored to a given trial's anticipated barriers/enablers. ${ }^{33}{ }^{35}$ We aimed to use this comprehensive framework to identify the breadth of anticipated and unanticipated (to the trial team) barriers and enablers to hypothetically participating in the first investigator-led Canadian CAR-T cell therapy trial for haematological malignancies. By using the TDF to identify anticipated barriers and enablers prior to trial launch, we aimed to inform strategies to support patients considering participation in the CAR-T cell therapy trial.

\section{METHODS}

We used the TDF to guide qualitative study design, data collection and analysis. ${ }^{36} 37$ We conducted qualitative semistructured interviews to identify potential barriers and enablers to participation in an early phase CAR-T cell therapy clinical trial and used the Consolidated Criteria for Reporting Qualitative studies to guide the reporting of this study. ${ }^{38}$

\section{Patient and public involvement in research}

Patient partners provided guidance and feedback on interview guide development and the interpretation of results as described below.

\section{Interview guide development}

We defined our target behaviour according to the Action, Actor, Context, Target, Time framework that specifies target behaviours according to "who needs to do what differently,' when, and in what context (see online supplemental file 1 for how the target behaviour was defined) ${ }^{39}$ We used the validated version of the TDF to develop interview questions and prompts to elicit views on factors that might impede or facilitate participation. ${ }^{33} 34$ Eligibility for the CAR-T trial was not assessed as part of this study. However, participants were asked to imagine they were eligible and to respond to hypothetical questions regarding potential barriers and enablers to trial participation. To ensure our questions were relevant and the phrasing was clear, we sought feedback from clinicians and piloted our interview guide with collaborating patient partners (TH and SS). The full interview guide is provided as online supplemental file 1 .

\section{Sampling strategy}

The planned CAR-T trial is being offered to patients with refractory or relapsed acute lymphocytic leukaemia, 
chronic lymphocytic leukaemia and B cell non-Hodgkin's lymphoma who have no other treatment options available. We opted to recruit adult patients who had been diagnosed with any type of haematological malignancy given that this patient population may be asked to participate in similar early phase immunotherapy cancer trials at some point in their illness trajectory and, therefore, serve as a useful analogue for identifying potential barriers and enablers to CAR-T therapy participation. Regardless, every effort was made to recruit patients who would likely be eligible for the proposed CAR-T trial. Prospective participants were referred to the study team by haematologists from two academic hospitals located in urban centres in Ontario and British Columbia (Canada). A national nonprofit organisation also distributed emails through their local chapters so that members of the non-profit who expressed interest in participating in an interview could contact the research team. We followed the $10+3$ rule to determine our target sample size and gauge whether we had collected enough data to delineate our conceptual themes while minimising participant burden. ${ }^{40}$

\section{Analysis}

Interviews were transcribed verbatim and analysed using the qualitative data analysis software NVivo Pro V.11. Data instances (ie, interview excerpts) were deductively coded into domains by three members of the research team (SA, GC and MF) according to existing guidelines. ${ }^{33} 3437$ SA and MF independently coded the first 10 interviews and GC and MF independently coded the last three interviews. Analysts met to discuss their coding strategies and resolve discrepancies. When analysts disagreed, they coded data instances into all domains for which an acceptable rationale was provided and noted what aspects of the data instance corresponded with identified domains. Double-coding was used to ensure our analyses did not exclude elements in the data due to a single analyst's interpretation.

We then conducted a directed content analysis using the TDF as the guiding framework. ${ }^{37}{ }^{41}$ Data instances within a given domain were inductively and deductively analysed for novel and expected domain specific content. A descriptive label or 'belief statement' was assigned to each data instance representing underlying beliefs, ideas and attitudes expressed by participants. Belief statements were then revised, compared and grouped into domainspecific subthemes based on similar qualities (eg, belief statements representing various concerns regarding treatment side effects). Belief statements and associated subthemes were further examined and grouped into broader within-domain categories where appropriate.

Counts were generated for data instances at the belief statement, subtheme and category level. Tables were generated showcasing categories, subthemes, belief statements, the number of participants that endorsed a specific belief and the frequency with which a belief appeared in the data set (see online supplemental file 2, for a comprehensive list of belief statements, subthemes, and frequency counts). We conducted a member check of our emerging analyses by providing a patient partner (TH) with a written summary of results (see online supplemental file 3). ${ }^{42}$ Feedback from TH informed our analysis of which beliefs were the most relevant to understanding participation in clinical trials. By considering the strength and relevance of beliefs, the presence of conflicting beliefs, and the frequency with which they occurred in the data set, we were able to identify key domains. ${ }^{37}$ Subthemes were compared across relevant domains to identify broad patterns in the data. Recurring themes and ideas that transcended key domains were synthesised into global themes representing the most important factors impacting the decision to participate in a CAR-T cell therapy trial.

\section{RESULTS}

\section{Interviews}

Interviews were collected between October 2017 and June 2018. The first 10 interviews were conducted by SA, a woman-identified research coordinator with a master's degree in health systems management, and the last three interviews were conducted by GC, a woman-identified research coordinator with a master's degree in psychology. Both interviewers were trained in using the TDF and received guidance and feedback from an expert in TDF methodology and behaviour change theory (JP). SA and GC introduced themselves as research staff and stated the general goals of the research (to identify barriers and enablers to trial participation) as part of the consent process. Interviews were conducted over the phone $(n=7)$ or in person $(n=6)$. Caregivers were present during four interviews. Interviews were audio recorded and ranged in length from 23 to $63 \mathrm{~min}$ (median=40 min). Written consent was obtained from every participant.

\section{Participants}

Thirteen people living with different types of haematological cancers (eight men and five women) participated in interviews. None of the participants who contacted the research team refused to participate or dropped out of the study. Most participants $(n=8)$ were recruited by their haematologists and five were identified by a non-profit organisation. Nine participants lived in Ontario and four lived in British Columbia. Participants ranged in age from 18 to 73 (median=56) and had been living with cancer from a few months to several years. Seven participants had a form of leukaemia. Two had acute lymphocytic leukaemia, one had chronic lymphocytic leukaemia and four had other diagnoses. Six participants had lymphoma with four specifying non-Hodgkin's lymphoma and three specifying B cell lymphomas. Eight participants were undergoing treatment at the time of the interview and five were in remission. Every patient had received at least one round of chemotherapy, half had received three or more rounds of chemotherapy, and three participants had undergone a bone marrow transplant. Two participants 
had prior experience participating in a clinical trial but none had experience participating in immunotherapy trials or early phase cancer trials. All participants were interviewed during the design phase of the planned CAR-T cell therapy trial.

\section{Saturation}

Data saturation occurs when no new themes emerge in the data. According to the $10+3$ rule, saturation is achieved when no new shared themes appear in the last three interviews. ${ }^{40}$ We did not find new subthemes in the last three interviews for 13 out of the 14 domains. A new subtheme was identified in the Reinforcement domain in the thirteenth interview, however, given that the reinforcement domain was not identified as a key domain, this is not likely to diminish credibility of the results presented.

\section{Key domains and global themes}

Six domains were identified as relevant to understanding barriers and enablers to participating in an early phase CAR-T cell therapy trial: Beliefs about Consequences, Knowledge, Social Influence, Intentions, Goals, and Environmental Context and Resources. Key domain subthemes were organised into four global themes. Themes are briefly described below and are then discussed in detail.

The first global theme, Navigating Choice in the Face of Uncertainty (domains: knowledge, beliefs about consequences, goals) characterises the dilemma of high stakes decision making as it applies to participating in an early phase CAR-T cell therapy trial. These considerations were heavily influenced by the second theme, Trusting the Experts (domain: social influence), where patients described the importance of reviewing trial options with their physicians before making a decision to participate. The third global theme, Ambivalent Intentions (domain: intention), represents the difficulty in arriving at a firm position, or intent, on whether or not to participate. The last global theme, Enabling Resources (domains: environmental context and resources, social influence), describes the material and social resources that may influence participation and enhance patient experiences during the trial. Table 1 presents subthemes, example belief statements and representative quotes for each global theme and associated domains.

\section{Global theme 1: navigating choice in the face of uncertainty}

Participants demonstrated varied levels of knowledge about CAR-T cell therapy. Some $(n=8)$ had heard about CAR-T cells from doctors, national organisations, and the news while others reported never having heard of them before the interview $(n=5)$. Regardless, most expressed a desire to know more about treatment safety $(\mathrm{n}=11)$ and efficacy $(\mathrm{n}=10)$, including remission and survival rates as compared with other treatment options. Participants wished to know more about trial logistics $(n=10)$ and agreed that receiving accessible trial information $(n=7)$ online $(n=4)$ and in written formats $(n=6)$ that could be shared with family and caregivers was important to them (domain: knowledge).
The need for information was born out of concerns over side effects $(n=7)$, including the risk of death $(n=2)$ (domain: beliefs about consequences). Other participants were more concerned with reducing the experience of pain and discomfort $(n=4)$ (domain: goals). One participant who had recently relapsed after receiving three rounds of chemotherapy indicated that they would prioritise quality of life over survival given their older age (domain: goals).

Despite these concerns, nine participants were motivated to participate in a clinical trial for the benefit of others though one participant indicated that altruism was not a motivating factor (domain: goals). Two explained their altruistic motivation as having 'nothing to lose but lots to gain'. Most participants were also motivated by the prospect of achieving progression-free survival $(n=10)$ or a cure $(n=5)$ despite having been informed that the purpose of an early phase CAR-T trial would be to assess safety (domains: beliefs about consequences, goals). Given the high risks and rewards associated with CAR-T cell therapy, a young woman who had experienced complications during chemotherapy and had recently recovered from a bone marrow transplant likened early phase trial participation to a game of 'Russian roulette' (domain: Beliefs about Consequences), emphasising the complexity and severity of deciding to participate in an early phase trial given the uncertain outcomes:

R: ... it's this like thing that could potentially cure me and it could be really good for me but also I could die from it, you know, it's freaky to think like oh this could cure me it's like Russian roulette. It's like this could be the bullet or this could not.

Participant \#11

\section{Global theme 2: trusting the experts}

Every participant $(\mathrm{n}=13)$ wished to hear about a CAR-T cell therapy trial directly from their haematologist or oncologist and indicated they valued their specialist's opinion (domain: social influences). When asked whose input they would want when considering participation, one participant responded:

R: My hematologist yeah. I mean they're like the group of seven, they're all a whole bunch of painters, you know, and they all sit together and discuss which I'm so fortunate. So yeah from the team from the haematology team that would be beneficial if it came from [them].

\section{Participant \#4}

Family members $(n=10)$, research staff $(n=7)$ and other healthcare providers (eg, family physicians, nurses) $(n=5)$ were also identified as influential persons. However, their input was secondary to the medical opinion of their specialist. Two participants indicated they trusted input from friends who were medical doctors the most (domain: social influences).

\section{Global theme 3: ambivalent intentions}

Most participants $(\mathrm{n}=11)$ wavered in their intentions suggesting they would participate if they knew they would benefit from doing so $(n=9)$, if they had few other treatment 
Table 1 Global themes, key domains, subthemes and example belief statements and quotes

$\begin{array}{lll}\begin{array}{l}\text { Relevant } \\ \text { domains }\end{array} \text { Subtheme Sample belief statements* } & \\ \end{array}$

Global theme 1: navigating choice in the face of uncertainty

Knowledge CAR-T cell therapy I did not know about CAR-T cells knowledge gaps before this interview. (5)

(potential barrier)

I would like to know more about

treatment efficacy. (10)

I would like to know more about the safety and side effects of CAR-T. (11)

I would like to know more about the logistics of participating in a CAR-T trial. (10)

Trial Information Delivery Methods

(enabler)

I would like accessible information. 'No to be frank with you I don’t really know very much [laugh] about that.' - Participant \#12

'I guess I would want them to sort of have a fairly good sense as to whether or not overall it was gonna be beneficial for me long-term or, you know, especially in comparison with what other treatment modalities are available to me.' - Participant \#8

'You know and possible side effects and what any side effects like you were saying and what if there's any back-up plan to, you know, like you say reversing those side effects. Or I know there's no guarantee about anything but just if there was a plan implemented to mitigate most or whatever could be done to reverse the side effects or treat the side effects appropriately and to know all the risks of doing the trial.' - Participant \#7

'I think that would depend on like what would be the logistics? I'm supposing that it would be done at or around the hospital but in other words is there something which could be done at hospital, at home? Is it all hospital? Depending on, on the nature of the tests and trials and so forth is it once/week or once every 3 weeks, you know those kinds of things. So, it would be important to know what the logistics would be to say, you know, can I manage this?' Participant \#13

I would like written trial information. (6)

I would like to get trial information online or from a website. (4)

$\begin{array}{ll}\begin{array}{l}\text { Beliefs about } \\ \text { Consequences }\end{array} & \begin{array}{l}\text { Treatment benefits } \\ \text { (enabler) }\end{array} \\ & \begin{array}{l}\text { Health benefits } \\ \text { (enabler) }\end{array}\end{array}$

\section{CAR-T cell therapy may be a more} tolerable alternative to chemo and BMTs. (6)

CAR-T cell therapy may help achieve remission or find a cure. (8)

Side effects and I am concerned about the potential

safety

(barrier)

side effects and safety of CAR-T. (7)

I'm concerned about the risk of death. (2)

Evaluating risk 'It's like Russian roulette' participating ' (barrier/enabler) is a high risk, high reward situation. (4) Surviving is important to me (selfpreservation) (10)

Achieving long-term remission or a cure is important to me (5)

Altruism (enabler)

Helping future generations is important to me. (9)

I am not motivated by altruistic considerations. (1)

Quality of life (enabler)
'I think paper and online both, but I think making the information as like I don't know striking a balance between being very detailed and still being like using accessible language. And I

'Just maybe like a book, I found like the transplant information books and everything very helpful even though it is explained to you. When a bunch of information like that is thrown at you, you don't really absorb all of it.' - Participant \#11

'Well websites are good when there's a lot of information.' - Participant \#8

'[laugh] If there's something that works as well or better than the strains of the chemotherapy well then it's an easy answer to hear that you want to see that.' -Participant \#3

'So if you can achieve remission and it just doesn't come back then, you know, that's to me that's a huge benefit and, like the chemo I have only been doing it for 5 months and it's taken a big toll on my body. And, you know, I'm way weaker than I was and way less energy and all that stuff. I still got another almost 2 years left of it. So yeah I guess that would be the biggest benefit being cured of leukemia without the long-term effects of chemo.' - Participant \#1

'Yeah it would be the level of advancement on that off switch. And the risk of, you know, how much work had been done on the safety I guess and the risk of dying or the other negative side effects.' - Participant \#1

'Because we're all different and this could work for a hundred people and kill one and I could be that one so I would be nervous about that.' - Participant \#2

'... it's this thing that could potentially cure me and it could be really good for me but also I could die from it, you know, it's freaky to think like this could cure me. It's like Russian roulette. It's like this could be the bullet or this could not.' - Participant \#11

'You know I'm still gonna, l'm fighting, I'm fighting tooth and nail and, you know, I'm gonna do whatever I can and hope that, you know, somewhere, sometime that we come up with something that will give a person a better survival rate and time, you know, so about time'. Participant \#7

'So if this is something that for instance has the possibility of them saying okay you go through this and maybe you won't need treatment again in the future or the chances of you needing treatment again in the future is reduced significantly then that would be, that would be a real positive for me. That would be something l'd want to know from the haematologists. ... So if, if a CAR T type of therapy if you can get a response like the so-called cure where you essentially have such a good remission that you're not worried about it anymore then that's something that's worthwhile.' - Participant \#8

'I think primarily to know that it's advantageous for people that have just been newly diagnosed. And of course survival, you know, l've got a grandson. Yeah, yeah but I think, you know, I think a big part of me just wants to see in that there's a benefit for people. I'm gonna cry now. For people, you know, in the future yeah. Not have to go through what I went through, yeah.' - Participant \#4

'I think the way in which it's framed to me the way in which it's presented. And like if it's presented as a way to help other people and to kind of, me to be an experiment essentially then I wouldn't feel so good about it.' - Participant \#5

'Whereas I was just like in the beginning before the chemo kicked in I was like oh yeah do whatever and then it was like okay I'm done with this we're gonna do what works and do what's gonna cause me the least pain and in the least amount of time. And then l'm gonna get out of here, you know.' - Participant \#11

'....there are a lot of people ... who will do the different things to stretch out life. And I think that's your choice, but I think quality of life is more important than the quantity, the actual timeline.' - Participant \#13 don't know l'm a very big fan of infographics and stats and percentages but obviously there wouldn't be that many that much of that because that's the whole point of doing the clinical trial to get that information.' - Participant \#5
Reducing the experience of pain and discomfort important to me. (4)

Quality of life is important to me. (3) 
Table 1 Continued

$\begin{array}{llll}\begin{array}{l}\text { Relevant } \\ \text { domains }\end{array} \quad \text { Subtheme } & \text { Sample belief statements* } & \text { Sample quotes }\end{array}$

Social Influence Haematologists and I trust my haematologist or oncologist 'My haematologist yeah. I mean they're like the group of seven, you know, they're all a whole oncologists and would want their opinion. (13) bunch of painters, and they all sit together and discuss which l'm so fortunate So yeah from (enabler) the haematology team that would be beneficial if it came from (hospital), yeah.' - Participant \#4

Healthcare system I can count on the healthcare system 'Because I when I talk to anybody my friends or anybody I always mention how I don't want (enabler) when things are tough. (11) to hear anybody complain about the (province) healthcare system because l've been, the care has been phenomenal for me.' - Participant \#10

Family and friends I would be the most influenced by my 'I think my medical doctor friends would be the most influential because they would inform me (enabler) doctor friends. (2) in how I would inform my husband too because he's not kind of medically oriented in how he thinks. So he would be relying heavily on me to explain sort of the risks and possible benefits and all that stuff. So yeah I think it would be my medical doctor friends.' - Participant \#1

\section{Global theme 3: ambivalent intentions}

Intentions No intent I would not participate in a CAR-T

clinical trial. (4)

'Hmm the safety of CAR T-cells yeah that might be the only reason that I might hesitate to participate, especially when I feel okay right now. I don't know if participating in that stage of the trial would benefit me.' - Participant \#9

Intent to participate I would participate in a CAR-T clinical 'I think we can make our voice known right now that yes, definitely we'd be interested in trial. (4)

Conditional intent I would be willing to try CAR-T cell therapy if I stand to gain from it. (9) participating.' - Participant \#3

I'm game to try it but at the same time it's like well if I'm not gonna be getting the drug say in like the what is it randomised ones where you're just getting something that looks like the drug. Would I really want to do something like that?' - Participant \#7

I would only participate in a CAR-T clinical trial if there were no other options. (6)

'I'd say if I didn't have any other decent options I guess like if I'm told after this next round whatever it is and it doesn't work if I'm told 'I'm sorry that's really all we can do for you,' I'd probably be very inclined to go into a study like that.' - Participant \#6

I would participate in a clinical trial if 'If my own haematologist felt that it was a worthwhile thing to try l'd probably consider it.' my doctor recommended it. (3)

I would rather participate in an efficacy trial than a safety trial. (2)

\section{Participant \#8}

'I think I'd honestly feel much less comfortable because safety I feel like is just it just has more negative conations with devastating side effects or minor side effects whereas (efficacy) is more associated with change for the better and growth and recovery. And yeah I would feel much less confident if it was phrased as testing the safety of this because then you think oh what's going to happen to me?' Participant \#5

$\begin{array}{lll}\text { Global theme 4: enabling resources } & \\ \begin{array}{lll}\text { Environmental } & \text { Enabling resources } & \text { Having parking and transportation } \\ \text { Context and } & \text { (enabler) } & \begin{array}{l}\text { paid for would help me attend } \\ \text { appointments. (11) }\end{array} \\ \text { Resources } & & \text { appont }\end{array}\end{array}$

Resources appointments. (11)

'Sure I mean obviously any benefit is more than welcomed. You know, as I've mentioned to you before, you know, the parking, of course, is a bit of a financial burden but, you know, we do have mechanisms in place. But yeah just knowing that you can come and go without that sort of, you know, burden would be beneficial for sure.' - Participant \#4

Having medication covered would be 'Yeah just coverage really it's just the only thing like I could possibly think of.' - Participant \#11 helpful. (3)

It would help to have child care services covered. (2)

It would help to cover caregiver costs (3)

'I mean if you've got people with kids that need childcare to be able to be part of the process well you do have to think of those, those aspects.' - Participant \#3

'Mm hmm definitely caregiver and transportation. I was lucky that I had my mom but if things had gone even remotely differently and she hadn't been able to get time off from work and if she hadn't had another alternative source of income which she does. She gets a pension from where she worked for a few decades. That's where we're from and that's where I was born and that is a very stabilising source of income in her life and in my family's life, but like if she hadn't had that then things would have been different.' - Participant \#5

would need to have accommodations 'I'm not sure just like is this a daily thing that is done or weekly or every 2 weeks that would covered to participate in a CAR-T make a difference. l'd have to look at housing and for my wife as well. She would travel with trial (2) me in all likelihood anyway. So housing would be a big thing. Yeah eat, of course, but you have to eat wherever you are so that's, you know, of.' - Participant \#6

I do not need any expenses covered. 'Yeah fortunately we're 10-15 min away from the hospital so it's not as if we're, we're travelling (6) from the country to be to be doing this so that's, that's one aspect it's, it's much easier for us so.' - Participant \#3

\begin{tabular}{|c|c|c|c|}
\hline \multirow[t]{2}{*}{ Social Influence } & \multirow[t]{2}{*}{$\begin{array}{l}\text { Desired social } \\
\text { supports } \\
\text { (enabler) }\end{array}$} & $\begin{array}{l}\text { Access to social workers, counselling, } \\
\text { and social resources. ( } 3 \text { ) }\end{array}$ & $\begin{array}{l}\text { 'I guess just greater follow-up with social workers in terms of getting access to employment } \\
\text { insurance for example and just ways to not use the system but just like use the resources that } \\
\text { are available to you to your advantage because the regular person doesn't know how to apply } \\
\text { for things like, you know, [non-profit offering financial assistance] what that even is. I didn't } \\
\text { know what that was until I was suddenly presented with all these pills that I had to take daily. } \\
\text { And so yeah definitely greater social work or whatever is involved in those kind of processes } \\
\text { in health.' - Participant \#5 }\end{array}$ \\
\hline & & $\begin{array}{l}\text { Having a health advocate or support } \\
\text { person is critical. (5) }\end{array}$ & $\begin{array}{l}\text { 'When things are less than life and death, no our system is a little too backlogged. Various } \\
\text { specialists are hard to get into unless you have somebody really fighting for you. You have } \\
\text { to be your own advocate in some cases but you almost have to have a little way in a little } \\
\text { backdoor or know somebody in some cases as well. Or you have to be willing to stand your } \\
\text { ground and make a fuss with certain people and it is kind of sad that way.' - Participant \#8 }\end{array}$ \\
\hline
\end{tabular}

*Numbers inbrackets indicate how many participants endorsed a specific belief statement. CAR, chimeric antigen receptor. 
options $(n=6)$, if their participation would help others $(n=4)$ and if their doctor asked them to participate $(n=3)$ (domain: intention).

I'd say if I didn't have any other decent options I guess like if I'm told after this next round whatever it is and it doesn't work if I'm told, I'm sorry that's really all we can do for you, I'd probably be very inclined to go into a study like that.

Participant \#6

Two participants suggested that they would be more willing to participate in an efficacy trial than a safety trial (domain: intention).

\section{Global theme 4: enabling resources}

Participants agreed that having participation-related expenses paid for would alleviate the financial burden associated with attending frequent appointments. Participants indicated parking and transportation $(n=11)$, medication $(n=3)$ and childcare services $(n=2)$ would be important resources to cover (domain: environmental context and resources). Caregiver costs $(n=3)$ and accommodations $(n=2)$ were important for those who anticipated having to travel long distances to participate in a trial (domain: environmental context and resources). One participant who routinely travelled several hours to receive treatment indicated that associated costs were a significant barrier and source of stress (domain: environmental context and resources). Conversely, some interviewees $(n=6)$ indicated that they would not need any resources covered because they lived close to the hospital and did not mind paying for transportation (domain: environmental context and resources).

Some participants wished for greater access to social workers and psychological counselling $(n=3)$ (domain: social influences). Others experienced difficulties accessing medical information and suggested a patient advocate would be helpful for presenting clinical trial options $(n=5)$ and for accessing specialists who may be difficult to contact (domain: social influences):

When things are less than life and death, no our system is a little too backlogged. Various specialists are hard to get into unless you have somebody really fighting for you.

$$
\text { Participant \#8 }
$$

\section{DISCUSSION}

We sought to identify barriers and enablers to hypothetically participating in a planned CAR-T cell therapy trial and found that participants were often ambivalent in their views. Participants expressed wanting to know more about CAR-T cell therapy and the logistics of the upcoming trial. They expressed concerns about potential side effects and the impact of trial participation on their quality of life. Many stated they were motivated by the promise of improved health despite stating that they understood the trial would primarily test for safety. Participants indicated that receiving information from specialists was valued and that having expenses covered and accessing social support might address financial and social barriers and improve the quality of their experience during the trial. These factors contributed to ambivalent intentions regarding trial participation. Though participants indicated that having no other treatment options would increase their likelihood of participation, the barriers and enablers identified in this study suggest that other factors likely contribute to patient decision making.

\section{Accessible information}

Accessible knowledge about CAR-T cell therapy that could be shared with loved ones was identified as a potential enabler suggesting trial details should be offered to prospective patients and their caregivers in multiple and accessible formats (eg, printed, verbal, online) to facilitate decisionmaking. Though information-based strategies do not always improve trial comprehension ${ }^{43}$ or recruitment rates, ${ }^{30}$ providing simplified consent forms and greater opportunities for patient-researcher conversations may increase patient comprehension levels. ${ }^{44}$ Using decision aids may be one way to support patients in making autonomous, informed decisions rather than deferring to physician recommendations. Ongoing research suggests that decision aids may increase comprehension of trial information and reduce decisional conflict and decisional regret without biassing participant decisions about whether to participate,${ }^{45-47}$ though more research is needed in this area. ${ }^{48} 49$

\section{Comparative data}

Participants in this study were motivated by the potential for health benefits and simultaneously expressed concerns over side effects. These findings resonate with Houghton et al's conceptual model which indicates that prospective randomised controlled trial participants will consider what they stand to gain or lose, altruistic motives, social influences and the burden of participation..$^{10}$ However, unique to the early phase cancer trial context is that patients often express therapeutic misconception (believing the intent of the trial is to benefit them) $)^{3150}$ and unrealistic optimism (believing they will experience better outcomes than others), ${ }^{18} 19$ posing ethical challenges for trialists. ${ }^{51}$

Consistent with existing guidance on developing decision aids for clinical trials, ${ }^{48}$ it will, thus, be important to present CAR-T cell therapy safety and efficacy findings with an emphasis on how safety and efficacy data compare to usual care and other available options. Though the threat of adverse events remains, the likelihood of experiencing adverse side effects may be comparable to treatments they have already endured. Likewise, reiterating the probability of experiencing a health benefit using non-biased language may enable prospective trial participants to make an informed decision rather than remaining shrouded in uncertainty or expecting health benefits when they cannot be guaranteed. Up-to-date systematic reviews provide an ideal source for describing the state of the evidence and provide a basis for developing accessible materials for patients and caregivers. ${ }^{3}$ Patients can then be engaged in comparative imaginings of future outcomes to aid with their decision-making process. ${ }^{32}$ 


\section{Role of haematologists and navigators}

Physicians have a clear role in helping patients formulate a decision regarding trial participation. However, given the multiple demands on physicians' time, patients could be directed to a patient navigator for trial information and social support. Patients who received support from patient navigators reported improved comprehension of clinical trials and a higher than average consent rate ${ }^{5253}$ Patient navigators may be a feasible strategy for meeting some patient needs during trial recruitment and participation. However, this warrants further research into the training necessary to ensure navigators do not inadvertently influence prospective trial participants (eg, by using personal narratives to convey information). ${ }^{5455}$

\section{Financial and social resources}

Participants indicated that having expenses like parking and transportation, accommodation and childcare covered would enable their participation. This is notable as parking costs have been identified as a significant source of financial toxicity that may impact cancer care. ${ }^{56}$ Social and financial resources should be allocated according to individual need to ensure that those who require greater assistance are not excluded from trial participation due to financial or social barriers. It will be important to remain transparent about what support is available to ensure equitable access to resources given the funding constraints of the trial.

\section{Strengths}

We used a comprehensive, evidence-based framework to ensure our findings on barriers and enablers cast a sufficiently wide net to capture likely impediments to participating in an early phase clinical trial. An advantage of the TDF is that key identified domains can be linked to evidence-based fit for purpose strategies to address barriers and enablers. ${ }^{32}$ Future research may seek to develop theory-driven strategies to better support patient decision making by mapping the barriers and enablers identified in this study to behaviour change techniques. ${ }^{57}$ Moreover, to the extent that other patient groups share similar barriers and enablers, similar strategies may serve to inform other similar trials.

We also prioritised patient voices by speaking with them in advance of the trial to ensure their perspectives were integrated in the trial design. Involving patient partners in this interview study helped to ensure our focus remained on patient identified priorities. Involving patient partners will continue as the trial progresses to ensure the research process remains relevant to patients. For example, patient partners have provided feedback on the trial protocol, consent forms, visual aides, and lay summaries, and in developing plans to organise a patient peer support panel. ${ }^{58}$

\section{Limitations}

As the trial had not yet begun, the aim of the present study was to identify anticipated challenges to inform efforts to optimise early phase trial conduct once the trial was launched. One limitation of this study is that we captured hypothetical barriers and enablers to participating in a planned CAR-T cell therapy trial. There is an opportunity for future research to document experienced barriers and enablers to participating in CAR-T cell therapy trials during the trials to assess any additional experienced barriers/enablers and whether the trial recruitment activities sufficiently addressed anticipated barriers/enablers identified here.

Second, those who are most likely to participate in research often experience fewer logistical barriers, prefer interviews over other types of designs, are altruistically motivated, and value research. ${ }^{59}$ This may be why nearly half of interview participants indicated they did not need any resources to participate in a trial, which may not fully reflect the realities of prospective trial participants. Nevertheless, a number of barriers were identified in these participants, indicating that even a motivated subset of participants with few resource barriers may face challenges in participating that are worth addressing in the design and conduct of the trial. More work is needed to understand the experiences of patients who face multiple socioeconomic barriers.

Finally, though participants varied in age, treatment course and length of time living with cancer, there were no discernable patterns indicating that barriers and enablers differed across these dimensions. We also did not collect information on race and ethnicity. Future research should aim to understand how these factors may impact participant decision making and trial participation experiences.

\section{CONCLUSION}

Using qualitative methods guided by a comprehensive framework enabled us to identify barriers and enablers before the trial, affording us the opportunity to develop trial specific strategies based on trial-specific barriers and enablers to improve recruitment procedures. Using the TDF may prove useful to inform the development of other early phase trials by ensuring that any unexpected barriers can be addressed to improve participation rates to early phase clinical trials and ultimately accelerate access to promising lifesaving therapies.

\section{Author affiliations}

${ }^{1}$ Blueprint Translational Research Group, Ottawa Hospital Research Institute, Ottawa, Ontario, Canada

${ }^{2}$ Clinical Epidemiology Program, Ottawa Hospital Research Institute, Ottawa, Ontario, Canada

${ }^{3}$ Department of Anaesthesiology and Pain Medicine, Ottawa Hospital General Campus, Ottawa, Ontario, Canada

${ }^{4}$ Blood and Marrow Transplant Program, Ottawa Hospital General Campus, Ottawa, Ontario, Canada

${ }^{5}$ School of Epidemiology and Public Health, University of Ottawa, Ottawa, Ontario, Canada

${ }^{6}$ Patient partner, Ottawa, Ontario, Canada

${ }^{7}$ ICES University of Ottawa, Institute for Clinical Evaluative Sciences, Ottawa, Ontario, Canada

${ }^{8}$ Genome Sciences Centre, BC Cancer, Vancouver, British Columbia, Canada ${ }^{9}$ Leukemia/BMT Program, Vancouver General Hospital, Vancouver, British Columbia, Canada

\section{Twitter Manoj M Lalu @manojlalu}

Acknowledgements We would like to thank the participants in this study, and their caregivers, who generously shared their time, experiences and wisdom with us. 
Contributors GC conducted three interviews, analysed data and drafted the manuscript. ML and JP designed the study and provided guidance and oversight for the conduct of the study and reporting of this manuscript. JP provided methodological expertise and leadership. SA designed interview guides, conducted interviews and analysed data. MF double coded data, provided feedback on analyses, and facilitated patient partner involvement. NK, DAF, HA, RAH and RB provided medical expertise and guidance on study design and interpretation of the results. NK, HA and RB assisted in identifying prospective participants. KT provided socioeconomic expertise and guidance on study design. JM provided feedback on study conduct and interpretation of results. TH and SS provided feedback on study design and results from the patient perspective. All authors provided feedback on multiple drafts of this manuscript.

Funding The conduct of this study was supported by a Clinical Social and Economic Impact (CSEI) grant from Biotherapeutics for Cancer Treatment (BioCanRx) (Grant reference number: FY17/CSEI4). BioCanRx is funded by the Government of Canada's Networks of Centres of Excellence. BioCanRx was not involved in any other aspect of the project, such as the design of the project's protocol, methods, analysis plan, the collection of data and analyses. ML is supported by The Ottawa Hospital Anesthesia Alternate Funds Association and the Scholarship Protected Time ProgramProgramme, Department of Anesthesiology and Pain Medicine, Ottawa.

Competing interests None declared.

Patient consent for publication Not required.

Ethics approval This study received ethics approval from the Ottawa Health Science Network Research Ethics Board (approval \#20170502-01H) and from the University of British Columbia - British Columbia Cancer Agency Research Ethics Board (approval \#H17-01472).

Provenance and peer review Not commissioned; externally peer reviewed.

Data availability statement All data relevant to the study are included in the article or uploaded as online supplemental information. The datasets generated and analysed during this study will not be made publicly available in order to protect the privacy and confidentiality of study participants. However, deidentified, aggregated data has been made available as online supplemental files.

Supplemental material This content has been supplied by the author(s). It has not been vetted by BMJ Publishing Group Limited (BMJ) and may not have been peer-reviewed. Any opinions or recommendations discussed are solely those of the author(s) and are not endorsed by BMJ. BMJ disclaims all liability and responsibility arising from any reliance placed on the content. Where the content includes any translated material, BMJ does not warrant the accuracy and reliability of the translations (including but not limited to local regulations, clinical guidelines, terminology, drug names and drug dosages), and is not responsible for any error and/or omissions arising from translation and adaptation or otherwise.

Open access This is an open access article distributed in accordance with the Creative Commons Attribution Non Commercial (CC BY-NC 4.0) license, which permits others to distribute, remix, adapt, build upon this work non-commercially, and license their derivative works on different terms, provided the original work is properly cited, appropriate credit is given, any changes made indicated, and the use is non-commercial. See: http://creativecommons.org/licenses/by-nc/4.0/.

\section{ORCID iDs}

Gisell Castillo http://orcid.org/0000-0002-0739-0393

Manoj M Lalu http://orcid.org/0000-0002-0322-382X

Dean A Fergusson http://orcid.org/0000-0002-3389-2485

\section{REFERENCES}

1 Maude SL, Teachey DT, Porter DL, et al. CD19-targeted chimeric antigen receptor T-cell therapy for acute lymphoblastic leukemia. Blood 2015;125:4017-23.

2 Salter Al, Pont MJ, Riddell SR. Chimeric antigen receptor-modified T cells: CD19 and the road beyond. Blood 2018;131:2621-9.

3 Grigor EJM, Fergusson D, Kekre N, et al. Risks and benefits of chimeric antigen receptor T-cell (CAR-T) therapy in cancer: a systematic review and meta-analysis. Transfus Med Rev 2019;33:98-110.

4 Davila ML, Riviere I, Wang X, et al. Efficacy and toxicity management of 19-28z CAR T cell therapy in B cell acute lymphoblastic leukemia. Sci Transl Med 2014;6:224ra25.

5 Biedrzycki BA. Decision making for cancer clinical trial participation: a systematic review. Oncol Nurs Forum 2010;37:E387-99.
6 Mills EJ, Seely D, Rachlis B, et al. Barriers to participation in clinical trials of cancer: a meta-analysis and systematic review of patientreported factors. Lancet Oncol 2006;7:141-8.

7 Canadian Cancer Research Alliance. Report on the state of cancer clinical trials in Canada. Toronto: Canadian Cancer Research Alliance, 2011.

8 Fayter D, McDaid C, Eastwood A. A systematic review highlights threats to validity in studies of barriers to cancer trial participation. $J$ Clin Epidemiol 2007;60:990.e1-990.e33.

9 Quinn GP, Koskan A, Wells KJ, et al. Cancer patients' fears related to clinical trial participation: a qualitative study. $J$ Cancer Educ 2012;27:257-62.

10 Houghton C, Dowling M, Meskell P, et al. Factors that impact on recruitment to randomised trials in health care: a qualitative evidence synthesis. Cochrane Database Syst Rev 2020;10:MR000045.

11 Unger JM, Cook E, Tai E, et al. The role of clinical trial participation in cancer research: barriers, evidence, and strategies. Am Soc Clin Oncol Educ Book 2016;35:185-98.

12 Bell JAH, Balneaves LG. Cancer patient decision making related to clinical trial participation: an integrative review with implications for patients' relational autonomy. Support Care Cancer 2015;23:1169-96.

13 Massett HA, Mishkin G, Rubinstein L, et al. Challenges facing early phase trials sponsored by the National cancer Institute: an analysis of corrective action plans to improve Accrual. Clin Cancer Res 2016;22:5408-16.

14 Ho J, Pond GR, Newman C, et al. Barriers in phase I cancer clinical trials referrals and enrollment: five-year experience at the Princess Margaret Hospital. BMC Cancer 2006;6:263.

15 Fu S, McQuinn L, Naing A, et al. Barriers to study enrollment in patients with advanced cancer referred to a phase I clinical trials unit. Oncologist 2013;18:1315-20.

16 van der Biessen DAJ, Cranendonk MA, Schiavon G, et al. Evaluation of patient enrollment in oncology phase I clinical trials. Oncologist 2013;18:323-9.

17 Dolly SO, Kalaitzaki E, Puglisi M. A study of motivations and expectations of patients seen in phase 1 oncology clinics: expectations of phase 1 oncology patients. Cancer 2016;122:3501-8.

18 Jansen LA, Mahadevan D, Appelbaum PS, et al. Variations in unrealistic optimism between acceptors and Decliners of early phase cancer trials. J Empir Res Hum Res Ethics 2017;12:280-8.

19 Jansen LA, Mahadevan D, Appelbaum PS, et al. Perceptions of control and unrealistic optimism in early-phase cancer trials. J Med Ethics 2018;44:121-7.

20 Nurgat ZA, Craig W, Campbell NC, et al. Patient motivations surrounding participation in phase I and phase II clinical trials of cancer chemotherapy. Br J Cancer 2005;92:1001-5.

21 Pawlowski J, Malik L, Mahalingam D. Advanced cancer patients' understanding and perceptions of phase I clinical trials. Cancer Invest 2015;33:490-5.

22 Comis RL, Miller JD, Colaizzi DD, et al. Physician-related factors involved in patient decisions to enroll onto cancer clinical trials. $J$ Oncol Pract 2009;5:50-6.

23 Albrecht TL, Blanchard C, Ruckdeschel JC, et al. Strategic physician communication and oncology clinical trials. J Clin Oncol 1999;17:3324-32.

24 Jenkins VA, Fallowfield LJ, Souhami A, et al. How do doctors explain randomised clinical trials to their patients? Eur $\mathrm{J}$ Cancer 1999;35:1187-93.

25 Jacobsen PB, Wells KJ, Meade CD, et al. Effects of a brief multimedia psychoeducational intervention on the attitudes and interest of patients with cancer regarding clinical trial participation: a multicenter randomized controlled trial. J Clin Oncol 2012;30:2516-21.

26 Meropol NJ, Wong Y-N, Albrecht T, et al. Randomized trial of a webbased intervention to address barriers to clinical trials. $J$ Clin Oncol 2016:34:469-78.

27 Guillot M, Asad S, Lalu MM, et al. So you want to give stem cells to babies? neonatologists and parents' views to optimize clinical trials. J Pediatr 2019;210:41-7.

28 Treweek S, Pitkethly M, Cook J, et al. Strategies to improve recruitment to randomised trials. Cochrane Database Syst Rev 2018;2:MR000013.

29 Preston NJ, Farquhar MC, Walshe CE, et al. Strategies designed to help healthcare professionals to recruit participants to research studies. Cochrane Database Syst Rev; 17 .

30 Kass NE, Sugarman J, Medley AM. An Intervention to Improve Cancer Patients' Understanding of Early-Phase Clinical Trials. IRB 2010;31.

31 Christopher PP, Appelbaum PS, Truong D, et al. Reducing therapeutic misconception: a randomized intervention trial in hypothetical clinical trials. PLoS One 2017;12:e0184224. 
32 Carey RN, Connell LE, Johnston M, et al. Behavior change techniques and their mechanisms of action: a synthesis of links described in published intervention literature. Ann Behav Med;384.

33 Michie S, Johnston M, Abraham C, et al. Making psychological theory useful for implementing evidence based practice: a consensus approach. Qual Saf Health Care 2005;14:26-33.

34 Cane J, O'Connor D, Michie S. Validation of the theoretical domains framework for use in behaviour change and implementation research. Implement Sci 2012;7:37.

35 Michie S, Johnston M, Francis J, et al. From theory to intervention: mapping theoretically derived behavioural determinants to behaviour change techniques. Appl Psychol 2008;57:660-80.

36 Phillips CJ, Marshall AP, Chaves NJ, et al. Experiences of using the theoretical domains framework across diverse clinical environments: a qualitative study. J Multidiscip Healthc 2015;8:139.

37 Atkins L, Francis J, Islam R, et al. A guide to using the theoretical domains framework of behaviour change to investigate implementation problems. Implement Sci 2017;12:77.

38 Tong A, Sainsbury P, Craig J. Consolidated criteria for reporting qualitative research (COREQ): a 32-item checklist for interviews and focus groups. Int J Qual Health Care 2007;19:349-57.

39 Presseau J, McCleary N, Lorencatto F, et al. Action, actor, context, target, time (AACTT): a framework for specifying behaviour. Implement Sci 2019;14:102.

40 Francis $\mathrm{JJ}$, Johnston M, Robertson $\mathrm{C}$, et al. What is an adequate sample size? Operationalising data saturation for theory-based interview studies. Psychol Health 2010;25:1229-45.

41 Hsieh H-F, Shannon SE. Three approaches to qualitative content analysis. Qual Health Res 2005;15:1277-88.

42 Graneheim UH, Lundman B. Qualitative content analysis in nursing research: concepts, procedures and measures to achieve trustworthiness. Nurse Educ Today 2004;24:105-12.

43 Hoffner B, Bauer-Wu S, Hitchcock-Bryan S, et al. "Entering a Clinical Trial: Is it Right for You?": a randomized study of The Clinical Trials Video and its impact on the informed consent process. Cancer 2012;118:1877-83.

44 Kass NE, Taylor HA, Ali J, et al. A pilot study of simple interventions to improve informed consent in clinical research: feasibility, approach, and results. Clin Trials 2015;12:54-66.

45 Juraskova I, Butow P, Bonner C, et al. Improving decision making about clinical trial participation - a randomised controlled trial of a decision aid for women considering participation in the IBIS-II breast cancer prevention trial. Br J Cancer 2014;111:1-7.
46 Politi MC, Kuzemchak MD, Kaphingst KA, et al. Decision AIDS can support cancer clinical trials decisions: results of a randomized trial. Oncologist 2016;21:1461-70.

47 Pathak S, George N, Monti D, et al. Evaluating adaptation of a cancer clinical trial decision aid for rural cancer patients: a mixed-methods approach. J Cancer Educ 2019;34:803-9.

48 Gillies K, Campbell MK. Development and evaluation of decision AIDS for people considering taking part in a clinical trial: a conceptual framework. Trials 2019;20:401.

49 Gillies K, Cotton SC, Brehaut JC, et al. Decision AIDS for people considering taking part in clinical trials. Cochrane Database Syst Rev 2015:CD009736.

50 Sulmasy DP, Astrow AB, He MK, et al. The culture of faith and hope. Cancer 2010;116:3702-11.

51 Halpern J, Paolo D, Huang A. Informed consent for early-phase clinical trials: therapeutic misestimation, unrealistic optimism and appreciation. J Med Ethics 2019;45:384-7.

52 Cartmell KB, Bonilha HS, Matson T, et al. Patient participation in cancer clinical trials: a pilot test of lay navigation. Contemp Clin Trials Commun 2016;3:86-93.

53 Nipp RD, Hong K, Paskett ED. Overcoming barriers to clinical trial enrollment. Am Soc Clin Oncol Educ Book 2019;39:105-14.

54 Bekker HL, Winterbottom AE, Butow P, et al. Do personal stories make patient decision AIDS more effective? A critical review of theory and evidence. BMC Med Inform Decis Mak 2013;13 Suppl 2:S9.

55 Winterbottom A, Bekker HL, Conner M, et al. Does narrative information bias individual's decision making? A systematic review. Soc Sci Med 2008;67:2079-88

56 Lee A, Shah K, Chino F. Assessment of parking fees at National cancer Institute-Designated cancer treatment centers. JAMA Oncol 2020;6:1295.

57 Michie S, Richardson M, Johnston M, et al. The behavior change technique taxonomy (V1) of 93 hierarchically clustered techniques: building an international consensus for the reporting of behavior change interventions. Ann Behav Med 2013;46:81-95.

58 Foster M, Fergusson DA, Hawrysh T, et al. Partnering with patients to get better outcomes with chimeric antigen receptor T-cell therapy: towards engagement of patients in early phase trials. Res Involv Engagem 2020;6:61.

59 Hepworth J, Robertson ARR, Jhunjhunwala A, et al. Cancer-Related psychosocial research: what are the perspectives of cancer care centre users on participation? Support Care Cancer 2011;19:1029-35. 